\title{
Phonology is fundamental in skilled reading: Evidence from ERPs
}

\author{
JANE ASHBY \\ Central Michigan University, Mount Pleasant, Michigan
}

\begin{abstract}
Research consistently indicates the importance of phonological processing in early reading development, yet the role of phonology in skilled reading is still not well understood. Two event-related potential (ERP) experiments investigated the nature and time course of phonological processing during skilled visual word recognition using a masked priming paradigm. Phonological syllable priming was examined by presenting prime-target pairs either with the same first syllable, or with one letter more or fewer. In this visually matched design, items like po\#\#-PONY and pon\#\#\#-PONDER appeared in the congruent condition. Conversely, pon\#-PONY and po\#\#\#\#-PONDER appeared in the incongruent condition. In both experiments, the magnitude of the first negative peak (N1) was reduced in the phonologically congruent condition as compared to the incongruent condition. This syllable congruency effect is the first neurophysiological evidence for phonological syllable activation in the initial moments of visual word recognition. The early time course of this activation indicates that suprasegmental phonological processing is fundamental to skilled reading.
\end{abstract}

The cognitive representation of speech sounds, or phonology, plays a central role in reading development. The behavioral index of these developing representations is phonological awareness, measured as the ability to segment the syllables and phonemes heard in speech (e.g., say /stop/ without the $/ \mathrm{s} /$ ). Although this indicator of phonological processing ability involves only spoken language, phonological awareness in kindergarten predicts reading achievement at the end of first grade, and phonologically based reading interventions precipitate gains in reading achievement (National Institute of Child Health and Human Development, 2000; Rayner, Foorman, Perfetti, Pesetsky, \& Seidenberg, 2001). Furthermore, phonological deficits are a primary factor in dyslexia and are the most common source of reading difficulties (Bradley \& Bryant, 1983; Morris et al., 1998).

Whereas phonological processes are widely considered to be important for the initial development of word reading skills, their role in skilled reading continues to be debated (Doctor \& Coltheart, 1980; Share, 1995; Shaywitz \& Shaywitz, 2005). Many behavioral studies have found evidence for word-level phonological activation during skilled reading (for recent reviews of this extensive literature, see Rastle \& Brysbaert, 2006; Van Orden \& Kloos, 2005). This research indicates that phonological effects appear consistently in naming experiments but inconsistently in lexical decision. The perceived task-dependent nature of phonological effects has fueled debate about the extent and importance of phonology in word recognition (e.g., Frost, 1998). The present study informs this debate by examining the nature and time course of phonological activation during passive word reading using a temporally sensitive measure (event-related potentials, or ERPs) and a visually matched design to minimize possible orthographic confounds.

Previous studies suggest that skilled readers represent suprasegmental phonological information (specifically, syllables) during word recognition (Ashby \& Rayner, 2004; Carreiras, Alvarez, \& de Vega, 1993; Carreiras \& Perea, 2002; Chen, Lin, \& Ferrand, 2003; Ferrand, Segui, \& Humphreys, 1997; Hutzler, Conrad, \& Jacobs, 2005; cf. Schiller, 2000). These studies found shorter fixation durations, lexical decision times, and naming latencies when targets (BALANCE) were preceded by an identical initial syllable (ba\#\#\#\#) rather than a syllable with one letter more or less (bal\#\#\#\#) - that is, a syllable congruency effect. Since these measures were based on the end point of word recognition, they did not reflect word recognition processes in real time. The present study used ERPs to provide a continuous measure of word recognition processes as they unfold.

Observing the time course of phonological processing is key to understanding its role in reading. Previous ERP studies have demonstrated phonological priming in visual word recognition using homophones, pseudohomophones, and orthographically matched nonhomophone primes (e.g., Grainger, Kiyonaga, \& Holcomb, 2006; Newman \& Connolly, 2004). These lexical level phonological effects appear between 250 and $500 \mathrm{msec}$ after word presentation. Such experiments place an upper limit on the time course of phonological processing, but they leave open the possibility of observing earlier sublexical phonological prim-

J. Ashby, jane.ashby@cmich.edu 


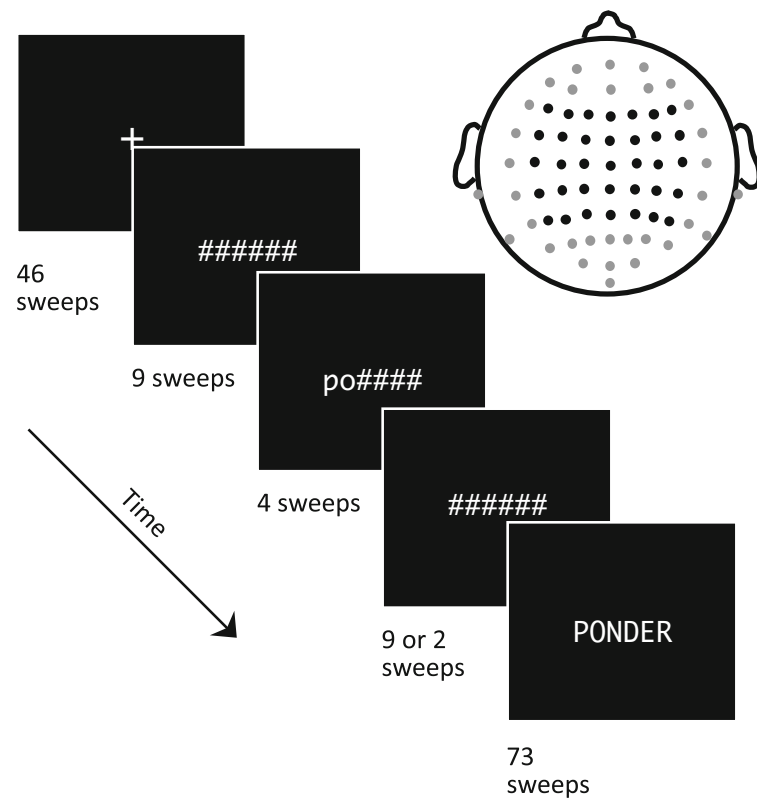

Figure 1. At top right is the approximate location of the 35 scalp electrodes selected a priori for analyses (black dots). The bottom left of the figure describes the masked priming paradigm.

ing effects. Further, they do not illuminate the nature of the phonological representation, since pseudohomophones prime any and all aspects of phonology (e.g., consonant and vowel segments, phonemes, and syllables).

Few ERP studies have examined sublexical phonological priming in isolated word recognition using masked priming. Ashby, Sanders, and Kingston (2009) conducted two experiments with a visually matched design that found subphonemic feature priming effects as early as $80 \mathrm{msec}$ posttarget. Ashby and Martin (2008) reported a preliminary syllable priming effect between 250 and $350 \mathrm{msec}$, but that experiment did not use a visually matched design. The present ERP study is the first to examine the time course of syllable processing using a visually matched design, such that the identical primes and targets appeared in both conditions.

The present study attempted to replicate the preliminary syllable effect in ERPs reported in Ashby and Martin (2008) using a visually matched design so that the same primes and targets appeared in both the incongruent and congruent conditions (Luck, 2005, p. 68). Participants silently read target words in a masked priming paradigm and made semantic decisions to filler items. Item pairs with different syllabification, such as PONY and PONDER, were matched for initial trigram. Each item was preceded by incongruent and congruent masked primes across participants. The same prime appeared in both conditions for a given participant (e.g., po\#\#-PONY and po\#\#\#\#-PONDER); thus, congruency was independent of the visual and orthographic characteristics of primes and targets. The masked priming paradigm allowed the presentation of advance word form information without a reader being consciously aware of it (see Figure 1). As the prime is not processed consciously, this paradigm is generally considered to tap the automatic processes used in word recognition (Forster \& Davis, 1984; Kinoshita \& Lupker, 2003; but see Norris \& Kinoshita, 2008). The principal difference between experiments was the prime-target interval. Experiment 1 used a $100-\mathrm{msec}$ interval consistent with the original study (Ashby \& Martin, 2008). The use of a 22-msec interval in Experiment 2 tested whether the early congruency effect in Experiment 1 would be repeated in a more typical masked-priming environment (e.g., Grainger et al., 2006).

The predictions are straightforward. If skilled readers activate suprasegmental phonological information, the congruency of the syllable information between prime and target should modulate ERPs and elicit different waveforms in the incongruent and congruent conditions. Early waveform modulation (i.e., before $200 \mathrm{msec}$ ) would indicate the involvement of phonology in fundamental aspects of the real-time process of word recognition, whereas later modulation would be consistent with existing evidence for phonology's later role in sentence integration processes (Rayner \& Pollatsek, 1989).

\section{METHOD}

\section{Participants}

Students at the University of Massachusetts were paid $\$ 18$ or received three experimental credits to participate in each experiment. Twenty right-handed, native English speakers ( 8 female, 12 male) with normal or corrected-to-normal vision participated in Experiment 1 . Eighteen similar students ( 8 female, 10 male) participated in Experiment 2.

\section{Procedure}

EEG was recorded continuously as participants read single words silently. A monitor running at $90 \mathrm{~Hz}$ (refresh rate, $11.1 \mathrm{msec}$ ) displayed words in cyan with a black background. ${ }^{1}$ E-Prime software (www.psnet.com) presented the materials and recorded manual responses. Participants initiated trials with a keypress. Each participant read every target word once, with half preceded by incongruent primes and half by congruent primes. Participants viewed any given target pair preceded by the identical masked prime (e.g., pon\#-PONY and pon\#\#\#-PONDER), with half of the items preceded by incongruent primes and half by congruent primes in a counterbalanced design (see Table 1). On half the filler items $(25 \%$ of the trials), participants got a visual prompt to make a semantic judgment via keypress (e.g., "Is it clothing?," "Does it fly?," "Is

Table 1

Counterbalancing of Materials for Experiments 1 and 2

\begin{tabular}{|c|c|c|c|c|}
\hline & 1st Mask & Prime & 2nd Mask & Target \\
\hline \multicolumn{5}{|c|}{ Counterbalancing Condition 1} \\
\hline Incongruent condition & \#\#\#\#\# & bas\#\# & \#\#\#\#\# & BASIN \\
\hline Congruent condition & \#\#\#\#\# & bas\#\#\# & \#\#\#\#\# & BASKET \\
\hline \multicolumn{5}{|c|}{ Counterbalancing Condition 2} \\
\hline Incongruent condition & \#\#\#\#\#\# & ba\#t & \#\#\#\#\#\# & BASKET \\
\hline Congruent conditiol & \#\#\#\# & ba\#\#\# & \#\#\#\#\# & BASIN \\
\hline
\end{tabular}


it bigger than a shoebox?") immediately after target presentation. Mean accuracy was 95\% and 96\% in Experiments 1 and 2, respectively. Each experiment was completed in one session of approximately $60 \mathrm{~min}$. The procedures in the two experiments differed only with respect to the display details and the duration of the primetarget interval. Experiment 1 presented trials in 14-point Courier and center justified in foveal view, whereas Experiment 2 presented trials in 10-point Monaco and left justified in foveal view (in both experiments, $2-3$ characters $=1^{\circ}$ of visual angle). Experiment 1 used a longer duration backward mask (100 msec), whereas Experiment 2 used a shorter duration mask $(22 \mathrm{msec})$. These parameters were varied to assess the sensitivity of any effect to changes in lowlevel visual information.

\section{Materials}

Participants read randomly presented target words (64 in Experiment 1 and a subset of 48 in Experiment 2). Pairs of targets with two versus three phonological segments in the initial syllable (e.g., $P O . N Y$ and $P O N . D E R$ ) were matched for initial trigram. Each type of target had a mean length of six letters. Because many words in English are ambisyllabic, syllabification was checked with the MRC Psycholinguistic Database (Wilson, 1988) and Merriam-Webster's Collegiate Dictionary (1995). Target trials were interspersed with 366 unrelated filler items that were also preceded by masked primes. One half of the filler primes were initial-syllable, and one half were full nonword primes. All filler targets were words.

\section{ERP Methods and Data Analysis}

EEG/EOG activity was recorded in DC from $64 \mathrm{Ag} / \mathrm{AgCl}$ active electrodes at $512 \mathrm{~Hz}$. EEG and EOG potentials were amplified by Biosemi Active-Two amplifiers. EEProbe software was used for signal processing (www.ant-neuro.com). The EEG for each participant was bandpass filtered at $0.16-30 \mathrm{~Hz}$ and referenced to averaged mastoids offline. After rejecting artifactual trials, the EEG was segmented into epochs from the onset of the forward mask to $700 \mathrm{msec}$ posttarget-onset. Individual ERP averages of the recorded, filtered voltages were baseline corrected for $82 \mathrm{msec}$ (Experiment 1) or $84 \mathrm{msec}$ (Experiment 2) during the forward mask preceding the prime. ${ }^{2}$

Individual averages were combined to produce grand averages. ${ }^{3}$ Preliminary analyses were guided by differences visible in the grand average waveform in three time windows: 100-120 msec, $140-160 \mathrm{msec}$, and $340-380 \mathrm{msec}$. Subsequent tests confirmed the onset and offset of each effect with further analyses of mean amplitudes measured in 20-msec windows $(60-700 \mathrm{msec})$ for each condition at 35 electrode sites selected a priori to create complete rows and columns that coded for electrode position (see Figure 1). Potentials in each window were subject to repeated measures ANOVAs that included four within-participants factors: prime-target congruency, target type ( 2 or 3 segment initial syllable), and electrode position (rows and columns) in a $2 \times 2 \times 5 \times 7$ design. Contrasts with more than one degree of freedom are reported with Greenhouse-Geisser corrected $p$ values. In order to avoid confounds arising from physical differences in the item pairs, only congruency effects that were evident across the full set of items - that is, main effects - are presented. The N1 was identified as the first negative peak visible after target onset in each experiment.

\section{RESULTS}

In Experiment 1 (see Figure 2), prime-target pairs with the same initial syllable (e.g., po\#\#-PONY) elicited a smaller N1 (140-160 msec) than targets preceded by a prime that had one more or one less letter (e.g., pon\#-PONY). The main effect of syllable congruency was significant across 28 electrode sites in the frontal, frontocentral, central, and centroposterior rows $[F(1,19)=4.45$, $p=.048]$, and barely missed significance across sites
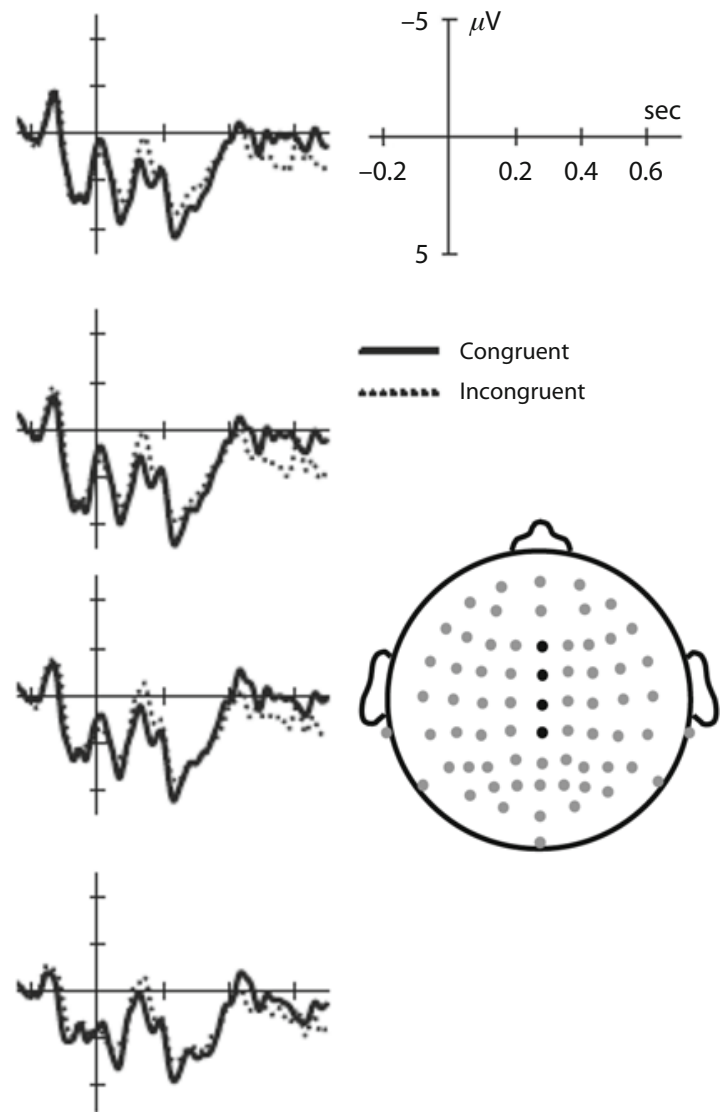

Figure 2. Sample midline electrodes from Experiment 1 show a reduced N1 elicited in the syllable-congruent condition (bold line) relative to the incongruent condition (dotted line). Target onset occurred at $0 \mathrm{msec}$. The recording sites of the displayed waveforms are described in the lower-right electrode layout (black dots).

in all five rows $[F(1,19)=4.29, p=.052]$. There was no interaction between rows and congruency, suggesting somewhat greater variability in potentials at posterior sites. No significant congruency effects appeared in other time windows.

In Experiment 2 (see Figure 3), targets in the syllablecongruent conditions elicited a smaller N1 (100-120 msec) than targets in the incongruent condition across electrodes in all rows $[F(1,17)=6.37, p=.022]$. In the 340 - to 380 -msec time window, a congruency effect appeared in the left three columns over the left hemisphere $[F(1,17)=$ $5.08, p=.04]$. In contrast to the earlier effect, potentials were more negative in the congruent condition than in the incongruent condition.

Omnibus ANOVAs tested for effects across experiments in each of the time windows. In the 100- to 120-msec time window, there was a main effect of congruency $[F(1,36)=$ $5.41, p=.03]$ and no experiment $\times$ congruency interaction $(F<1)$. The main effect of congruency across experiments is consistent with the congruency effect in Experiment $2(100-120 \mathrm{msec})$ reported above, and it suggests that a nonsignificant trend toward a congruency effect started in this earlier time window for Experiment 1. In the 140- to $160-\mathrm{msec}$ time window, there was no indication of a main 

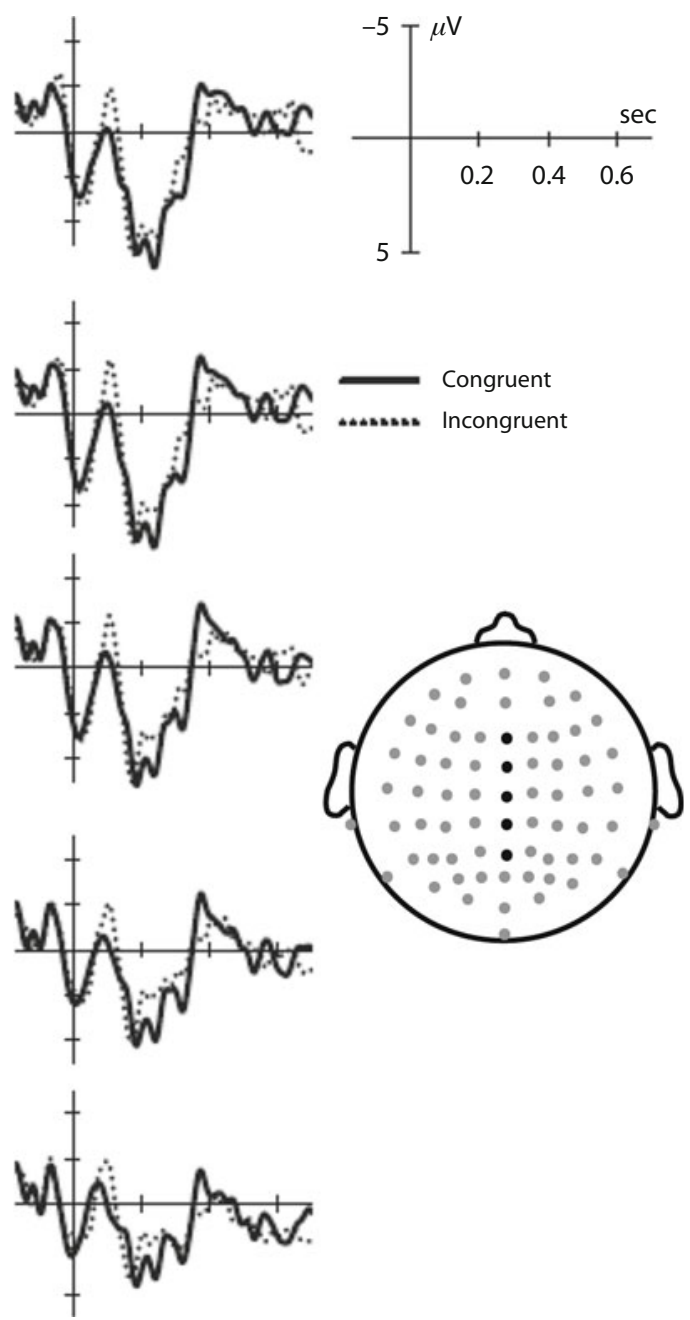

Figure 3. Sample midline electrodes from Experiment 2 show a reduced N1 elicited in the syllable-congruent condition (bold line) relative to the incongruent condition (dotted line). Target onset occurred at $0 \mathrm{msec}$. The recording sites of the displayed waveforms are described in the lower-right electrode layout (black dots).

effect of congruency $(F<1)$. The experiment $\times$ congruency interaction failed to reach significance $[F(1,36)=$ $2.34, p=.14]$. However, this may primarily indicate a lack of power because of the limited item set. This interpretation is consistent with the significant congruency effect found in Experiment 1 (140-160 msec) without any accompanying evidence for a trend toward a congruency effect in this time window in Experiment $2(F<0.5)$.

\section{DISCUSSION}

The most striking finding in these experiments was reduced N1 amplitudes to targets preceded by syllablecongruent primes. The phonological congruency effect onset as early as $100 \mathrm{msec}$ when words were read silently, and this indicated that fast phonological processing occurs during skilled word recognition. This is the earliest evidence for suprasegmental phonological activation yet observed, and the use of visually identical materials in the congruent and incongruent conditions eliminates the possibility that this effect is orthographic in nature. Backward mask duration may have affected the timing of the N1 across experiments, but the congruency effect appeared aligned with the N1 in each case. Therefore, the present effect indicates that skilled readers quickly activate phonological syllable information en route to word identification.

The early syllable congruency effect coincides with other word recognition effects that are beginning to appear in the ERP literature (e.g., Ashby et al., 2009; Hauk et al., 2006) and clearly indicates that suprasegmental phonological representations can exist very early in the process of recognizing a written word. However, the import of finding syllable congruency effects in the N1 portion of the waveform is unclear at this time. It seems unlikely that the present congruency effect involves the classic N1 attention component, given the posterior scalp distribution of the attention effect and its somewhat later onset (Mangun \& Hillyard, 1995). Interestingly, the distribution and timing of the present early congruency effect appears more similar to the auditory N1 effect reported by Sanders and Neville (2003). Sanders and Neville compared the ERPs elicited during the processing of word-initial and wordmedial spoken syllables. Their data indicate increased N1 amplitudes to word-initial syllables, and they attribute this effect to increased processing demands in the wordinitial condition. Similarly, the incongruent conditions in the present study would require increased processing as a reader shifts from one suprasegmental representation to another because of prime-target incongruency.

The present syllable congruency effects appeared earlier than the phonological effects that Grainger et al. (2006) observed in a masked priming ERP study with transposed letter and pseudohomophone primes, and earlier than the syllable effect reported in Ashby and Martin (2008). Two factors may have contributed to the detection and replication of the early congruency effect in the present experiments. First, the use of identical materials in both conditions minimized any variance that the visual properties of unmatched primes and targets might contribute to the early ERP waveform (Luck, 2005). Second, the consistent location of the present manipulation (i.e., at letter position 3) minimized variance in the physiological response that can "smear" early effects (Penolazzi, Hauk, \& Pulvermüller, 2007). Therefore, it appears that the combination of a visually matched design and a consistent manipulation across experimental items permitted the detection of an early phonological congruency effect.

Careful readers may notice a shift in the timing of the N1 across experiments, which is evident from a visual comparison of Figures 2 and 3, but not statistically significant. Although this shift should be interpreted cautiously, seeing a decreased N1 latency to targets presented at shorter prime-target intervals is consistent with a preliminary masked priming study that manipulated the prime-target interval within participants (Holcomb \& Grainger, 2007, Figure 1). Whereas the prime-target interval may have had 
an effect on when target processing was initiated, it did not affect the phonological nature of the initial processing.

The masked priming paradigm is traditionally considered to tap the automatic processes used in word recognition (Forster \& Davis, 1984). However, Norris and Kinoshita (2008) demonstrated that masked priming effects are easily modulated by changing the task instructions. Their paper underscores the importance of considering how the task environment in the present experiments might have influenced the results. Participants were instructed to read the words silently and to make occasional semantic categorization decisions to filler items. The semantic task encouraged reading for meaning, and the use of several semantic categories minimized the tendency to prepare a response after reading each word. To perform the task, participants needed to have the word in short-term memory, as would be expected during normal sentence reading. Therefore, the observed syllable effects appeared without any overt phonological task, which suggests that skilled readers typically represent phonological information early in the word-recognition process.

Several eye-movement studies have reported syllable congruency effects during the silent reading of sentences (e.g., Ashby, 2006; Ashby \& Rayner, 2004). Target words were read faster when the parafoveal preview had the same initial syllable than when the preview had one letter more or less. The present study provides converging electrophysiological evidence that skilled readers activate suprasegmental phonology when reading isolated words. In addition, this study indicates when deflections in the ERP waveform register the processing of foveal syllable information. The previous eye-movement studies differ from the present experiments in several dimensions, yet the parafoveal preview and masked priming paradigms share one characteristic: Both present advance word form information that readers are unaware of processing. The eye-movement studies indicate that phonological overlap between this advance information and the consciously processed word can facilitate word recognition and reduce word reading time. The present ERP experiments indicate that syllable information is activated early. Therefore, it appears that phonology may have an early role in speeding skilled word recognition when readers are accessing a specific lexical entry.

The present syllable congruency effect complements the phonological feature priming effect reported in Ashby et al. (2009). In that study, prime-target pairs with congruent final consonant voicing (fak-FAT and faz-FAD) elicited less negative brain potentials than did pairs with incongruent voicing (faz-FAT and fak-FAD). This feature congruency effect onset around $80 \mathrm{msec}$ in both experiments, regardless of the prime-target interval. This contrasts with the present syllable effects, which coincided with the timing of the N1. Together, these studies provide neurophysiological evidence that skilled readers activate syllables as well as subphonemic feature information during the initial moments of visual word recognition. Therefore, early phonological representations are multilayered and complex.
In summary, these experiments are the first to report an early syllable congruency effect in ERPs using a visually matched design, which indicates that skilled readers activate suprasegmental phonological information early in word recognition. The present study complements previous research reporting early phonological feature congruency effects (Ashby et al., 2009), and provides converging neurophysiological evidence for a fundamental role for phonological activation in skilled reading. The accumulating evidence for the early activation of subphonemic features and syllables suggests that the fast processing of complex phonological information characterizes the end point of reading development. This implies a developmentally continuous role for phonological processes in reading across the lifespan and thus helps to account for the persistent effect of phonological deficits on reading achievement beyond the childhood years.

\section{AUTHOR NOTE}

NIH Grant HD051700 supported this research. I thank Charles E. Clifton, Lisa D. Sanders, and Keith Rayner for comments on previous versions of this article, and Laura Giffin for help collecting the data Correspondence concerning this article should be addressed to J. Ashby, Department of Psychology, 137 Sloan Hall, Central Michigan University, Mt. Pleasant, MI 48859 (e-mail: jane.ashby@cmich.edu).

\section{REFERENCES}

Ashby, J. (2006). Prosody in skilled silent reading: Evidence from eye movements. Journal of Research in Reading, 29, 318-333.

Ashby, J., \& Martin, A. E. (2008). Prosodic phonological representations early in visual word recognition. Journal of Experimental Psychology: Human Perception \& Performance, 34, 224-236.

Ashby, J., \& RAYNER, K. (2004). Representing syllable information during silent reading: Evidence from eye movements. Language \& Cognitive Processes, 19, 391-426.

Ashby, J., Sanders, L. D., \& Kingston, J. (2009). Skilled readers begin processing phonological features by $80 \mathrm{msec}$ : Evidence from ERPs. Biological Psychology, 80, 84-94.

BRADLEY, L., \& BRYANT, P. D. (1983). Categorizing sounds and learning to read - a causal connection. Nature, 301, 419-421.

Carreiras, M., Alvarez, C. J., \& de Vega, M. (1993). Syllable frequency and visual word recognition in Spanish. Journal of Memory \& Language, 32, 766-780.

Carreiras, M., \& Perea, M. (2002). Masked priming effects with syllabic neighbors in a lexical decision task. Journal of Experimental Psychology: Human Perception \& Performance, 28, 1228-1242.

Chen, J.-Y., Lin, W.-C., \& Ferrand, L. (2003). Masked priming of the syllable in Mandarin Chinese speech production. Chinese Journal of Psychology, 45, 107-120.

Doctor, E. A., \& Coltheart, M. (1980). Children's use of phonological encoding when reading for meaning. Memory \& Cognition, 8 , 195-209.

Ferrand, L., Segui, J., \& Humphreys, G. W. (1997). The syllable's role in word naming. Memory \& Cognition, 25, 458-470.

Forster, K. I., \& DAVIs, C. (1984). Repetition priming and frequency attenuation in lexical access. Journal of Experimental Psychology: Learning, Memory, \& Cognition, 10, 680-698.

Frost, R. (1998). Toward a strong phonological theory of visual word recognition: True issues and false trails. Psychological Bulletin, 12, 71-99.

Grainger, J., Kiyonaga, K., \& Holcomb, P. J. (2006). The time course of orthographic and phonological code activation. Psychological Science, 17, 1021-1026.

Hauk, O., Patterson, K., Woollams, A., Watling, L., PulvermülLER, F., \& Rogers, T. T. (2006). [Q:] When would you prefer a SOSSAGE to a SAUSAGE? [A:] At about $100 \mathrm{msec}$. ERP correlates of 
orthographic typicality and lexicality in written word recognition Journal of Cognitive Neuroscience, 18, 818-832.

Holcomb, P. J., \& Grainger, J. (2007). Exploring the temporal dynamics of visual word recognition in the masked repetition priming paradigm using event-related potentials. Brain Research, 1180, 39-58.

Hutzler, F., Conrad, M., \& Jacobs, A. M. (2005). Effects of syllable frequency in lexical decision and naming: An eye-movement study. Brain \& Language, 92, 138-152.

Kinoshita, S., \& LUPKer, S. J. (2003). Masked priming: The state of the art. New York: Psychology Press.

LUCK, S. J. (2005). An introduction to event-related potential technique. Cambridge, MA: MIT Press.

Mangun, G. R., \& Hillyard, S. A. (1995). Mechanisms and models of selective attention. In M. D. Rugg \& M. G. H. Coles (Eds.), Electrophysiology of mind: Event-related brain potentials and cognition (pp. 40-85). Oxford: Oxford University Press.

Merriam-Webster (1995). Merriam-Webster's collegiate dictionary (10th ed.). Springfield, MA: Author.

Morris, R. D., Stuebing, K. K., Fletcher, J. M., Shaywitz, S. E., Lyon, G. R., Shankweiler, D. P., ET AL. (1998). Subtypes of reading disability: Variability around a phonological core. Journal of Educational Psychology, 90, 347-373.

National Institute of Child Health and Human Development (2000). Teaching children to read: An evidence-based assessment of the scientific research literature on reading and its implications for reading instruction (NIH Publication No. 00-4754). Washington, DC: National Institutes of Health.

Newman, R. L., \& Connolly, J. F. (2004). Determining the role of phonology in silent reading using event-related brain potentials. Cognitive Brain Research, 21, 94-105.

Norris, D., \& Kinoshita, S. (2008). Perception as evidence accumulation and Bayesian inference: Insights from masked priming. Journal of Experimental Psychology: General, 137, 434-455.

Penolazzi, B., Hauk, O., \& Pulvermüller, F. (2007). Early semantic context integration and lexical access as revealed by event-related brain potentials. Biological Psychology, 74, 374-388

RAstLe, K., \& Brysbaert, M. (2006). Masked phonological priming effects in English: Are they real? Do they matter? Cognitive Psychology, 53, 97-145.

Rayner, K., Foorman, B., Perfetti, C., Pesetsky, D., \& Seiden-
BERG, M. (2001). How psychological science informs the teaching of reading. Psychological Science in the Public Interest, 2, 31-73.

Rayner, K., \& Pollatsek, A. (1989). The psychology of reading. Englewood Cliffs, NJ: Prentice Hall.

Sanders, L. D., \& Neville, H. J. (2003). An ERP study of continuous speech processing I. Segmentation, semantics, and syntax in native speakers. Cognitive Brain Research, 15, 228-240.

Schiller, N. O. (2000). Single word production in English: The role of subsyllabic units during phonological encoding. Journal of Experimental Psychology: Learning, Memory, \& Cognition, 26, 512-528.

Share, D. L. (1995). Phonological recoding and self-teaching: Sine qua non of reading acquisition. Cognition, $\mathbf{5 5}, 151-218$.

Shaywitz, S. E., \& ShaYwitz, B. A. (2005). Dyslexia (specific reading disability). Biological Psychiatry, 57, 1301-1309.

VAN ORden, G. C., \& KloOs, H. (2005). The question of phonology and reading. In M. J. Snowling \& C. Hulmes (Eds.), The science of reading (pp. 61-78). Malden, MA: Blackwell.

WILSON, M. D. (1988). The MRC psycholinguistic database: Machine readable dictionary, Version 2. Behavior Research Methods, Instruments, \& Computers, 20, 6-11.

\section{NOTES}

1. Because of equipment changes, the materials were presented at a $75-\mathrm{Hz}$ refresh rate for 4 participants in Experiment 1 and Experiment 2.

2. In Experiment 1, these electrodes were interpolated using a spherical-spline formula available in EEProbe software: $\mathrm{F} 3$ (for 2 participants) and F5, F6, FC3, FC4, FC5, C3, CP2, CP3, and CP6 for 1 participant each. In Experiment 2, the following electrodes were interpolated using the same formula: CP3 (for 2 participants) and F5 and F6 (for 1 participant each).

3 . The congruent and incongruent grand averages in Experiment 1 comprised 553 data points $(M=27.7, S D=2.6)$ and 508 data points $(M=25.4, S D=3.1)$, respectively. Experiment 2 grand averages comprised 351 data points $(M=19.5, S D=2.8)$ and 354 data points $(M=$ $19.6, S D=2.9)$

(Manuscript received January 28, 2009; revision accepted for publication September 10, 2009.) 